ACCepted for publication in ApJL; 2016 March 27

Preprint typeset using $\mathrm{LAT}_{\mathrm{E}} \mathrm{X}$ style emulateapj v. 5/2/11

\title{
RADIO FLARING FROM THE T6 DWARF WISEPC J112254.73+255021.5 WITH A POSSIBLE ULTRA-SHORT PERIODICITY
}

\author{
Matthew Route ${ }^{1,2,3}$ \& Alexander Wolszczan ${ }^{1,2}$ \\ Accepted for publication in ApJL; 2016 March 27
}

\begin{abstract}
We present new results from a continuing $5 \mathrm{GHz}$ search for flaring radio emission from a sample of $\mathrm{L}$ and $\mathrm{T}$ brown dwarfs, conducted with the 305-m Arecibo radio telescope. In addition to the previously reported flaring from the T6.5-dwarf 2MASS J10475385+212423, we have detected and confirmed circularly polarized flares from another T6-dwarf, WISEPC J112254.73+255021.5. Although the flares are sporadic, they appear to occur at a stable period of 0.288 hours. Given the current constraints, periods equal to its second and third subharmonic cannot be ruled out. The stability of this period over the 8-month timespan of observations indicates that, if real, it likely reflects the star's rapid rotation. If confirmed, any of the three inferred periodicities would be much shorter than the shortest, 1.41hour rotation period of a brown dwarf measured so far. This finding would place a new observational constraint on the angular momentum evolution and rotational stability of substellar objects. The detection of radio emission from the sixth $\sim 1000 \mathrm{~K}$ dwarf further demonstrates that the coolest brown dwarfs and, possibly, young giant planets, can be efficiently investigated using radio observations at centimeter wavelengths as a tool.
\end{abstract}

\section{INTRODUCTION}

Radio observations of ultracool dwarfs (UCDs; Kirkpatrick et al. 1997)) address important issues in the physics of low-mass stars, brown dwarfs, and planets. The most commonly discussed problem is the generation and topology of the magnetic fields in fully-convective, low-mass stars and brown dwarfs (Donati et al. 2006 Morin et al. 2010). Because these objects are cool, rapid rotators, the usual magnetic activity indicators provided by $\mathrm{H} \alpha$ and X-ray emission become inefficient, and the Zeeman splitting based technique does not work, due to the rotational broadening of spectral lines employed in this method (Gizis et al. 2000; Stelzer et al. 2006, Reiners \& Basri 2007; McLean et al. 2012). On the other hand, radio detections, especially of the electron cyclotron maser (ECM) driven, rapid bursts of emission, enable a direct measurement of the lower bound to the local magnetic field strength (Treumann (2006), and references therein), provide means to measure stellar rotation (Williams \& Berger 2015), and diagnose the conditions of the magnetosphere - ionosphere interaction (Nichols et al. 2012). In addition, modeling of the time-frequency (dynamic) spectra of these bursts provides information on the UCD magnetic field topology (Hess et al. 2011 Kuznetsov et al. 2012; Lynch et al. 2015).

Another problem that can be addressed using radio detection as a tool is the angular momentum evolution of UCDs (Zapatero Osorio et al. 2006; Reiners \& Basri 2008). Rotation period measurements of young brown dwarfs in clusters demonstrate that these objects lose

\footnotetext{
${ }^{1}$ Department of Astronomy and Astrophysics, the Pennsylvania State University, 525 Davey Laboratory, University Park, PA 16802, alex@astro.psu.edu

2 Center for Exoplanets and Habitable Worlds, the Pennsylvania State University, 525 Davey Laboratory, University Park, PA 16802

${ }^{3}$ Current Address: Research Computing, Information Technology at Purdue, Purdue University, 155 S. Grant St., West Lafayette, IN 47907, mroute@purdue.edu
}

very little of their initial angular momentum as they evolve (Scholz et al. 2015). Measurements of the periodic radio bursts generated by the coolest, old dwarfs probe the late stages of their rotational evolution. The advantage of using ECM-generated radio flaring for period measurements over optical/near-infrared photometry is that their precision is not dependent on cloud-induced intensity variations. For example, the apparent radio flaring period of a rapidly-rotating, M9-type UCD, TVLM $513-46546$, shows only a $\sim 0.03 \%$ variation around the average value of $7054.468 \mathrm{~s}$ calculated over a 7 -year baseline (Wolszczan \& Route 2014). In contrast, the measurability and precision of photometrically-derived periods critically depend on the ratio of the period itself to the timescale of cloud evolution (Heinze \& Metchev 2015).

The late L, T, and Y-type dwarfs detectable in the radio can also be treated as proxies for studies of the magnetic properties of young, massive exoplanets, assuming, for example, the magnetic field scaling predictions discussed by Reiners \& Christensen (2010). Although attempts to detect low-frequency radio emission from hot Jupiters generated by the stellar wind - planetary magnetosphere interaction have not been successful (e.g. Lazio et al. (2010), and references therein), GHzfrequency measurements of hot exoplanets discovered by direct imaging at optical wavelengths (e.g. (Marois et al. 2008) ) may offer a practical alternative in the form of detections of ECM radio bursts similar to those generated by the coolest UCDs (Route \& Wolszczan 2012; Kao et al. 2016).

Motivated by these considerations, we have been searching for polarized radio flaring from these objects with the Arecibo radio telescope. Our first survey of $33 \mathrm{UCDs}$ at $5 \mathrm{GHz}$ has resulted in the first discovery of a radio emitting T-dwarf, the T6.5 UCD, 2MASS J10475385+212423 (J1047+21; Route \& Wolszczan 2012, 2013). Follow-up Jansky Very Large Array (JVLA) observations detected quiescent radio emission from this object (Williams et al. 2013), confirmed its 
flaring component (Williams \& Berger 2015, Kao et al. 2016), and produced a measurement of the 1.77-hr rotation period of the star (Williams \& Berger 2015). In addition, guided by the fact that $J 1047+21$ is also a weak $\mathrm{H} \alpha$ emitter, Kao et al. (2016) have detected quiescent and flaring radio emission from four out of five $\mathrm{H} \alpha$ and optical/infrared variability-selected L and T-type UCDs.

Here, we report the Arecibo detection of another Tdwarf, WISEPC J112254.73+255021.5 (J1122+25), in the course of the second part of our unbiased survey of late L and T-type UCDs. The most distinguishing characteristic of this new radio-emitting UCD is its tentatively established ultra-fast rotation period. If confirmed, it will become by far the shortest one measured for any UCD.

Our observations, the detection of flares from $\mathrm{J} 1122+25$, their characteristics, and a tentative measurement of the UCD's rotation period are described in Section 2. Further discussion of these results and our conclusions are given in Section 3. The entire Arecibo UCD survey will be described in a forthcoming paper.

\section{DETECTION OF FLARING FROM WISEPC $\mathrm{J} 112254.73+255021.5$}

WISEPC J112254.73+255021.5 was discovered in the course of the Wide-field Infrared Survey Explorer survey (WISE; Kirkpatrick et al. 2011). Medium resolution near-infrared spectroscopy using the SpeX on NASA Infrared Telescope Facility (IRTF) led to the classification of the object as a T6 brown dwarf, with a spectrophotometric distance estimate of 16.9 pc. Nearby M5 field star LHS 302 has similar distance and proper motion to that of J1122+25, but due to its low mass and a wide projected separation of $\sim 4500 \mathrm{AU}$, the objects are unlikely to be physically bound.

The continuing L and T- spectral type dwarf survey with the Arecibo radio telescope uses the instrumental setup and data analysis method discussed in detail by Route \& Wolszczan (2012, 2013). J1122+25 has been detected on 2013 May 8 as a broadband, left-hand circularly polarized (LCP), 1.5 mJy flare. Four additional LCP flares have been identified in the data taken on 2013 December 27 and 31, and 2014 January 4, during a weeklong confirmation run. The 2013 December 27 event, shown in Fig. 1 as the most prominent example of the J1122+25 flaring, consists of two $100 \%$ LCP bursts, occurring $\sim 1100 \mathrm{~s}$ apart, with peak fluxes of $\sim 3$ mJy and $\sim 2.5$ mJy, respectively, at the full, $0.1 \mathrm{~s}$ resolution. An additional 10 hours of observations made in 2015 May and June, non-simultaneously covering the 3-4 $\mathrm{GHz}$ and $4-5 \mathrm{GHz}$ bands, have not produced any additional detections. A search of the "14Dec17" release of the FIRST radio catalog found no radio sources within the half-power beam width of the telescope, with a detection limit of $0.92 \mathrm{mJy}$. The nearest sources are the star J112253.4+255250.2 at a separation of 148.8 arcsec, and J112247.6+254736.9, an unknown object 184.3 arcsec away with peak fluxes of $1.19 \mathrm{mJy}$ and $4.08 \mathrm{mJy}$, respectively, at $1.4 \mathrm{GHz}$. These sources are located well outside of the Arecibo $\sim 1$ arcmin beam at $5 \mathrm{GHz}$.

\subsection{Flare Characteristics}

$\mathrm{J} 1122+25$ is only the second radio emitting T-dwarf identified in an unbiased survey, after our detection of flaring from J1047+21 (Route \& Wolszczan 2012). $\mathrm{J} 1122+25$ emits broadband, short duration, 30 - $120 \mathrm{~s}$ pulses, characterized by a rapid, variable frequency drift (50-800 $\left.\mathrm{MHz} \mathrm{s}^{-1}\right)$ and left circular polarization ranging from $15 \%$ to $100 \%$. These characteristics are typical of ECM emission generated by electrons moving in the magnetic fields of rapidly rotating UCDs (Burgasser \& Putman 2005; Hallinan et al. 2006, 2008, Berger et al. 2009; Route \& Wolszczan 2012 Williams \& Berger 2015 Williams et al.2015). Similar to J1047+21, the emission is clearly sporadic with only five pulse detections in 29 hours of observations. However, as observed by Williams \& Berger (2015), J1047+21 does undergo periods of activity, during which several varying, consecutive pulses can be detected.

The shortest measurable timescale of intensity fluctuations in the flares can be used to estimate an upper limit to the source size and a lower limit to its brightness temperature. We have computed a noise-corrected autocorrelation function (ACF) of the strongest, December 27 flare sampled at the original, $0.1 \mathrm{~s}$ resolution, and integrated over the interference free parts of the total bandpass, $\Delta \nu=1 \mathrm{GHz}$ (Fig. 2). The ACF shows a clear change of slope around the $\sim 2$ s lag, indicating the presence of intensity fluctuations in the flare on a similar timescale. As the flare rapidly drifts in frequency at $d \nu / d t \sim 50-800 \mathrm{MHz} \mathrm{s}^{-1}$, this timescale most likely represents intensity variations that are faster than our 0.1 s resolution, but they are smeared by frequency drifting to $\Delta \nu(d \nu / d t)^{-1} \sim 1.25-2 \mathrm{~s}$. This places an upper limit of $\sim 0.4 \mathrm{R}_{J}$ on the size of the emitting region, and leads to the source's brightness temperature estimate, $\mathrm{T}_{b} \geq 4 \times 10^{11} \mathrm{~K}$.

The high degree of circular polarization of the $\mathrm{J} 1122+25$ flares and the above $\mathrm{T}_{b}$, which is close to the $\mathrm{T}_{b} \sim 10^{12} \mathrm{~K}$ Compton limit of compact radio sources (Kellerman \& Pauliny-Toth 1969), make it entirely feasible to assume that the ECM mechanism is responsible for their generation. In this case, the star's local magnetic field strength, $B$, can be estimated from the corresponding cyclotron frequency, $\nu_{c}=2.8 \times 10^{6} \mathrm{~B}$ (Gauss), where $\nu_{c}=5.2 \times 10^{9} \mathrm{~Hz}$, represents the upper end of the $1 \mathrm{GHz}$ receiver bandpass. Because the observed emission appears to extend beyond that frequency for all five flares, the cutoff frequency of emission has yet to be measured, and the computed $B \gtrsim 1.8 \mathrm{kG}$ must be treated as a lower limit to the actual magnetic field strength of the star.

\subsection{Periodicity in the J1122+25 Flaring?}

We have investigated the possibility that, despite their sporadic character, the flares may be periodic. If all five flares appear in phase with the dwarf's rotation period, it is obviously constrained by the $\sim 1100$ s separation of the two December 27 events (Fig. 1). To refine this period estimate and test whether the five flares are indeed phase-connected, we have used a Monte Carlo (MC) approach in which trial periods have been randomly drawn from a 15 to $20 \mathrm{~min}$. interval, and fitted to flare timesof-arrival (TOAs) with the TEMPC ${ }^{5}$ modeling code in a manner described in detail by Wolszczan \& Route (2014).

\footnotetext{
${ }^{2}$ Information available at http://tempo.sourceforge.net/
} 
The modeling process is complicated by the high variability of the flare profiles, thus making it impossible to unambiguously determine the phase reference points for TOA measurements. Therefore, in our procedure, we have included a random selection of TOAs from windows which were centered on the flares and spanned the full widths of flare profiles at the $10 \%$ flux density level. TEMPO was used to fit 2000 trial periods to each of the 500 sets of randomly-selected, five flare TOAs. Surprisingly, we have easily obtained a phaseconnected timing solution with a new, improved period of $1035.7881 \pm 0.0008 \mathrm{~s}$ and a post-fit $\mathrm{rms}$ residual, $\sigma \approx 0.86$ s. Phase alignment of the five flares at the best-fit period is shown in Fig. 3. The same period is obtained with the flare TOAs fixed at the approximate centers of the profiles, but the post-fit $\sigma$ becomes $\sim 10 \mathrm{~s}$, which reflects the TOA uncertainty due to the changing flare morphology (see also (Wolszczan \& Route 2014)).

As the result of period modeling based on only five data points must be treated with caution, we have checked its statistical significance with a MC simulation in which we have randomly drawn $5 \times 10^{5}$ sets of five time-tags of samples from the five, 10-minute, 667 -data point observing windows containing flares (Figs 1,3), and used TEMPO to fit the measured best-fit period to these simulated arrival times. By counting the post-fit $\sigma \leq 10 \mathrm{~s}$ values from these fits, we have computed a $0.01 \%$ false alarm probability (FAP) for our period measurement of the $\mathrm{J} 1122+25$ flares. For a much more restrictive, $\sigma \leq 1$ $\mathrm{s}$ criterion, the FAP becomes vanishingly small.

Another possibility is that the two, apparently consecutive, December 27 flares were generated by different activity regions in the magnetosphere, and only one of them was rotational phase-connected to the remaining three events. Such behavior has been observed in other well-studied, periodically radio emitting UCDs, such as TVLM 513 and 2MASS J07464256+2000321 (Lynch et al. (2015), and references therein). We have therefore conducted MC tests in the manner described above by removing either of these two flares from the data set. The best-fit period for the four flares with the first December 27 one removed is $1032.6988 \pm 0.0008 \mathrm{~s}$ with $\sigma=0.16$ s. Not surprisingly, this is very close to the $1035 \mathrm{~s}$ period calculated for the five flare case. The data are also very well fitted with the approximately third subharmonic of that period, $3107.519 \pm 0.002 \mathrm{~s}$ and $\sigma=0.82 \mathrm{~s}$. When the second flare is excluded, the best-fit period is $2053.1398 \pm 0.0005, \sigma=0.22 \mathrm{~s}$, expectably close to twice the shortest period, which necessarily produces the same fit. The FAP values for the two fits, using the $\sigma \leq 10 \mathrm{~s}$ criterion, are $0.2 \%$ and $0.02 \%$, respectively. As discussed above, it is important to remember that the tiny, formal TEMPO errors associated with the best-fit periods are much smaller than the actual, more realistic uncertainties of $\sim \pm 15$ s resulting from the flare profile variability and the associated ambiguity in determining the actual TOAs. These uncertainties have been estimated from the scatter of the best-fit periods with $\sigma \leq 10 \mathrm{~s}$ around the one with the lowest $\sigma$ value.

Finally, we have folded all the available data not containing the five flares, modulo the best-fit periods, to check for the possible presence of low-level, periodic emission. This analysis has not produced any obvious detection, down to the $3 \sigma$ level of $\sim 30 \mu \mathrm{Jy}$. We conclude that both the available observational evidence and the FAP estimates suggest that one of the three, obviously harmonically-related periodicities in the $\mathrm{J} 1122+25$ flaring over the period of 8 months is very likely to be real, but additional, extensive, possibly multi-wavelength observations are needed to verify this result.

\section{DISCUSSION AND CONCLUSIONS}

Our detection of radio flaring from the T-dwarf $\mathrm{J} 1122+25$, reported in this paper, significantly adds to the small group of five late-L and T-dwarfs previously known to display this type of activity (Route \& Wolszczan 2012, Kao et al. 2016, Williams et al. 2015). The $J 1122+25$ flares are LCP with brightness temperatures approaching $10^{12} \mathrm{~K}$, indicating their ECM origin. In this case, the local magnetic field strength must exceed 1.8 $\mathrm{kG}$, in agreement with similar measurements of other radio detected UCDs (Burgasser \& Putman 2005; Hallinan et al. 2006, 2008, Berger et al. 2009, Route \& Wolszczan 2012: Williams et al. 2015).

Clearly, the most interesting aspect of our radio detection of $\mathrm{J} 1122+25$ is the tantalizing possibility that the flares from this UCD occur at a period that may be as short as $\sim 17$ minutes. The shortest rotation periods derived from the photometric variability of UCDs at optical/near-infrared wavelengths are 1.41 and 1.55 hours, for the T6.5 dwarf 2MASS J22282889-4310262 (Clarke et al. 2008) and the T7 dwarf 2MASS J005019943322402 (Metchev et al. 2015), respectively. An even shorter, but never confirmed periodicity of $16.5 \mathrm{~min}$ has been reported for the T4.5 dwarf 2M 0559-1404 (Koen 2004). Bailer-Jones \& Mundt (2001) and Zapatero Osorio et al. (2003) have observed a varying, 30-46.4 min periodicity in the young, M8.5 dwarf S Ori 45, but, obviously, even if real, it could not be interpreted in terms of stellar rotation, because of its variability.

In principle, the rotational breakup period of an old brown dwarf, $P_{0}=2 \pi\left(R^{3} /(G M)\right)^{1 / 2}$, could be as short as $\sim 20$ min, using the standard value of $R$, which is the Jupiter radius, $R_{J}=7 \times 10^{7} \mathrm{~m}$, and the mass, $M \leq 80 \mathrm{M}_{J}$. Assuming $P_{0}=1035 \mathrm{~s}$, the shortest of the three possible periods of the $\mathrm{J} 1122+25$ flares, one needs $\mathrm{R} \leq 0.9 \mathrm{R}_{J}$ to ensure that $M \leq 80 M_{J}$. Taking into account a significant oblateness of the star induced by rapid rotation, and using the Darwin-Radau relationship (Barnes \& Fortney 2003 Sengupta \& Marley 2010), the oblateness of a rotating star is given in terms of its polar and equatorial radii, $\mathrm{R}_{p}$ and $\mathrm{R}_{e}$, as:

$$
f=1-\frac{R_{p}}{R_{e}}=\frac{\Omega^{2} R}{g}\left[\frac{5}{2}\left(1-\frac{3 K}{2}\right)^{2}+\frac{2}{5}\right]^{-1},
$$

where $\Omega$ is the angular rotational velocity of the star, $\mathrm{K}=\mathrm{I} /\left(\mathrm{MR}^{2}\right)$ ranges from 0.261 to 0.205 for the polytropic indices $n=1$ and $n=1.5$, respectively, and I is the moment of inertia. Assuming the observed value of $\Omega=6.1 \times 10^{-3}$ $\mathrm{s}^{-1}, \mathrm{n}=1.5$ for high surface gravity, the corresponding breakup oblateness, $f=0.38$ (James 1964), and $\mathrm{R}=0.8$ $\mathrm{R}_{J}$, we get $\log (\mathrm{g}) \sim 5.5$. This, together with constraints provided by the dwarf's temperature of $T_{e} \sim 1000 \mathrm{~K}$ (Kirkpatrick et al. 2011), and the evolutionary models of Burrows et al. (2001), gives $\mathrm{M} \geq 60 \mathrm{M}_{J}$ and a lower limit of $\sim 1$ Gyr to the age of the star. 
Of course, the two longer periods produce correspondingly less extreme characteristics of the dwarf. For example, for $\mathrm{P}_{0}=2053 \mathrm{~s}$ and $\mathrm{R}=\mathrm{R}_{J}$, the Darwin-Radau approximation gives $\log (\mathrm{g}) \geq 5$, which leads to $\mathrm{M} \geq 35 \mathrm{M}_{J}$ for $\mathrm{T}_{e}=1000 \mathrm{~K}$. If any of the three measured periods is real, all these estimates make $\mathrm{J} 1122+25$ an ultra-rapidly rotating, massive UCD with the radius falling well within the theoretical range for dwarfs older than 1 Gyr.

Despite the plausibility of the existence of sufficiently evolved dwarfs rotating at periods $\leq 1$ hour, based on the investigations of the angular momentum loss in the evolving substellar objects (see Bouvier et al. (2014), for a review), none have been detected so far. Whereas objects rotating close to their breakup periods may be rare, this situation could also be caused by observational biases. However, several ground and space-based surveys of $\mathrm{L}$ and $\mathrm{T}$ dwarfs conducted in the past had sufficiently high sensitivity and cadence of photometric measurements to be able to detect such periodicities (e.g. Metchev et al. (2015), and references therein). On the other hand, photometric fluctuations of the UCDs generated by cloud dynamics can dramatically blur their rotation-induced variability, even for the shortest periods observed so far (Radigan et al. 2012, Apai et al. 2013, Heinze \& Metchev 2015). The same could happen for objects with rotational periods $\leq 1$ hour, if the "weather" related variations on such the short timescales are still feasible.

Ideally, assuming any of the three possible periods discussed above, and a quasi-steady emission source, flares from J1122+25 should be detectable at least once during each, typically $\sim 2$-hour long observing session. Flaring from this dwarf is clearly sporadic, but, as in the case of the first radio detected T-dwarf, J1047+21 (Route \& Wolszczan 2012), it could occasionally undergo periods of higher activity (Williams \& Berger 2015). The sporadic appearance of flares trom $\mathrm{J1122+25}$ and a few other radio-emitting $\mathrm{T}$-dwarfs detected so far is most likely related to variations in the flow of electrons into the magnetic field needed to power the observed, ECM-generated flaring. Given the star's largely neutral, field-decoupled atmosphere, Mohanty et al. (2002), Schrijver (2009), and Nichols et al. (2012) have argued that, like in the cases of Jupiter and Saturn, the power needed to create such currents in the magnetosphere is extracted from rotation. In particular, Nichols et al. (2012) propose that such currents could be created through magnetic field reconnection at the boundary between the closed and the open field lines, where the magnetosphere opens up to the interstellar medium. As the conditions in this transition region are expected to be highly variable, it would naturally explain the intermittent character of the observed emission.

Of course, more work is needed to confirm the J1122+25 flaring period reported in this paper. This includes a study of its photometric variability and, if possible, a measurement of the $v \sin i$ value, which, if the rotation period reported here is real, is likely to be much above $100 \mathrm{~km} \mathrm{~s}^{-1}$. More attempts to detect radio flaring from the dwarf are also important. Finally, it would be of interest to verify if $\mathrm{J} 1122+25$ is a detectable $\mathrm{H} \alpha$ emitter and/or it exhibits any enhanced variability at optical/infrared wavelengths. If so, it would further strengthen the evidence presented by Kao et al. (2016) that the best candidates for detectable radio emission among the coolest UCDs are the ones that exhibit one or both of these properties.

\section{ACKNOWLEDGEMENTS}

MR acknowledges support from the Center for Exoplanets and Habitable Worlds, which is supported by the Pennsylvania State University, and the Eberly College of Science. The Arecibo Observatory is operated by SRI International under a cooperative agreement with the National Science Foundation (AST-1100968), and in alliance with Ana G. Méndez-Universidad Metropolitana, and the Universities Space Research Association. This paper has made use of NASA's Astrophysics Data System. 


\section{REFERENCES}

Apai, D., Radigan. J., Buenzli, E., et al. 2013, ApJ, 768, 121

Bailer-Jones, C. A. L., \& Mundt, R. 2001, A\&A, 367, 218

Barnes, J. W., \& Fortney, J. 2003, ApJ, 588, 545

Berger, E., Rutledge, R. E., Phan-Bao, N., et al. 2009, ApJ, 695, 310

Bouvier, J., Matt, S. P., Mohanty, S., et al. 2014, in Protostars and Planets VI, ed. H. Beuther et al. (Tucson, AZ: Univ. Arizona Press), 43

Burgasser, A. J., \& Putman, A. E. 2005, ApJ, 626, 486

Burrows, A., Hubbard, W. B., Lunine, J. I., \& Liebert, J. 2001, RvMP, 73, 719

Clarke, F. J., Hodgkin, S. T., Oppenheimer, B. R., Robertson, J., \& Haubois, X. 2008, MNRAS, 386, 2009

Donati, J.-F., Forveille, T., Collier Cameron, A., et al. 2006, Science, 311, 633

Gizis, J. E., Monet, D. G., Reid, I. N., Kirkpatrick, J. D., Liebert, J., \& Williams, R. J. 2000, AJ, 120, 1085

Hallinan, G., Antonova, A., Doyle, J. G., et al. 2006, ApJ, 653, 690

Hallinan, G., Antonova, A., Doyle, J. G., et al. 2008, ApJ, 684, 644

Heinze, A. N., \& Metchev, S. 2015, in Proc. of the Conference Held at Lowell Observatory, Cool Stars, Stellar Systems and the Sun: 18th Cambridge Workshop, ed. G. van Belle, \& H. C. Harris (Flagstaff, AZ: Lowell Observatory), 501, https://www.dropbox.com/sh/5fg67vejtkbhkxy/ AAC6EBwyVaRHPqbpufMUVzVTa?dl $=0$

Hess, S. L. G., \& Zarka, P. 2011, A\&A, 531, A29

James, R. A. 1964, ApJ, 140, 552

Kao, M. M., Hallinan, G., Pineda, J. S., et al. 2016, ApJ, 818, 24

Kellerman, K. I. \& Pauliny-Toth, I. I. K. 1969, ApJL, 155, L71

Kirkpatrick, J.D., Beichman, C. A., \& Skrutskie, M. F., 1997, ApJ, 476, 311
Kirkpatrick, J.D., Cushing, M. C., Gelino, C. R., et al. 2011, ApJS, 197, 19

Koen, C. 2004, MNRAS, 354, 378

Kuznetsov, A. A., Doyle, J. G., Yu, S., et al. 2012, ApJ, 746, 99

Lazio, T. J. W., Carmichael, S., Clark, J., et al. 2010, AJ, 139, 96

Lynch, C., Mutel, R., \& Güdel, M. 2015, ApJ, 802, 106

Marois, C., Macintosh, B., Barman, T., et al. 2008, Sci, 322, 1348

McLean, M., Berger, E., \& Reiners, A. 2012, ApJ, 746, 23

Metchev, S. A., Heinze, A., Apai, D., et al. 2015, ApJ, 799, 154

Mohanty, S., Basri, G., Shu, F., \& Allard, F. 2002, ApJ, 571, 469

Morin, J., Donati, J.-F., Petit, P., et al. 2010, MNRAS, 407, 2269

Nichols, J. D., Burleigh, M. R., Casewell, S. L., Cowley, S. W. H., Wynn, G. A., Clarke, J. T., \& West, A. A. 2012, ApJ, 760, 59

Radigan, J., Jayawardhana, R., Lafrenière, D., Artigau, É., Marley, M., \& Saumon, D. 2012, ApJ, 750, 105

Reiners, A., \& Basri, G. 2007, ApJ, 656, 1121

Reiners, A., \& Basri, G. 2008, ApJ, 684, 1390

Reiners, A., \& Christensen, U. R. 2010, A\&A, 522, A13

Route, M., \& Wolszczan, A. 2012, ApJL, 747, L22

Route, M., \& Wolszczan, A. 2013, ApJ, 773, 18

Scholz, A., Kostov, V., Jayawardhana, R., \& Mužić, K. 2015 ApJL, 809, L29

Schrijver, C. J. 2009, ApJL, 699, L148

Sengupta, S., \& Marley, M. S. 2010, ApJL, 722, L142

Stelzer, B., Micela, G., Flaccomio, E., Neuhäuser, R., \&

Jayawardhana, R. 2006, A\&A, 448, 293

Treumann, R. A. 2006, A\&AR, 13, 229

Williams, P. K. G., Berger, E., \& Zauderer, B. A. 2013, ApJL, 767, L30

Williams, P. K. G., \& Berger, E. 2015, ApJ, 808, 189

Williams, P. K. G., Berger, E., Irwin, J., Berta-Thompson, Z. K., \& Charbonneau, D. 2015, ApJ, 799, 192

Wolszczan, A., \& Route, M. 2014, ApJ, 788, 23

Zapatero Osorio, M. R., Caballero, J. A., Béjar, V. J. S., \& Rebolo, R. 2003, A\&A, 408, 663

Zapatero Osorio, M. R., Martín, E. L., Bouy, H., Tata, R., Deshpande, R., \& Wainscoat, R. A. 2006, ApJ, 647, 1405 


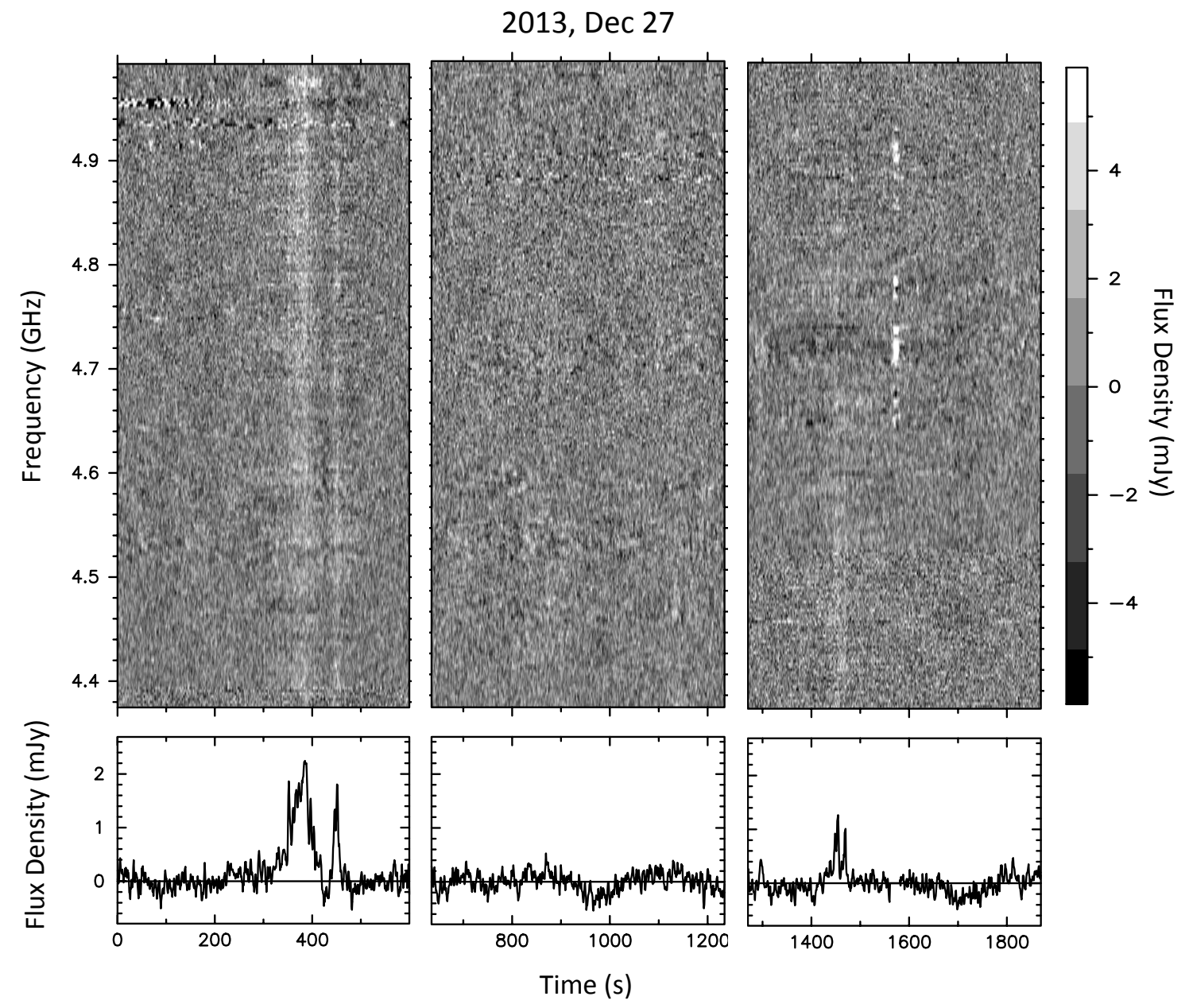

Figure 1. Dynamic spectra (top) and average time profiles (bottom) of the two LCP flares from J1122+25 displayed at the time and frequency resolution of $2.7 \mathrm{~s}$ and $2.5 \mathrm{MHz}$, respectively. The $35 \mathrm{~s}$ gaps between the adjacent scans are times spent on calibration. A series of spikes at $\sim 1600 \mathrm{~s}$, centered at $\sim 4.75 \mathrm{GHz}$, is due to interference of a terrestrial origin. 

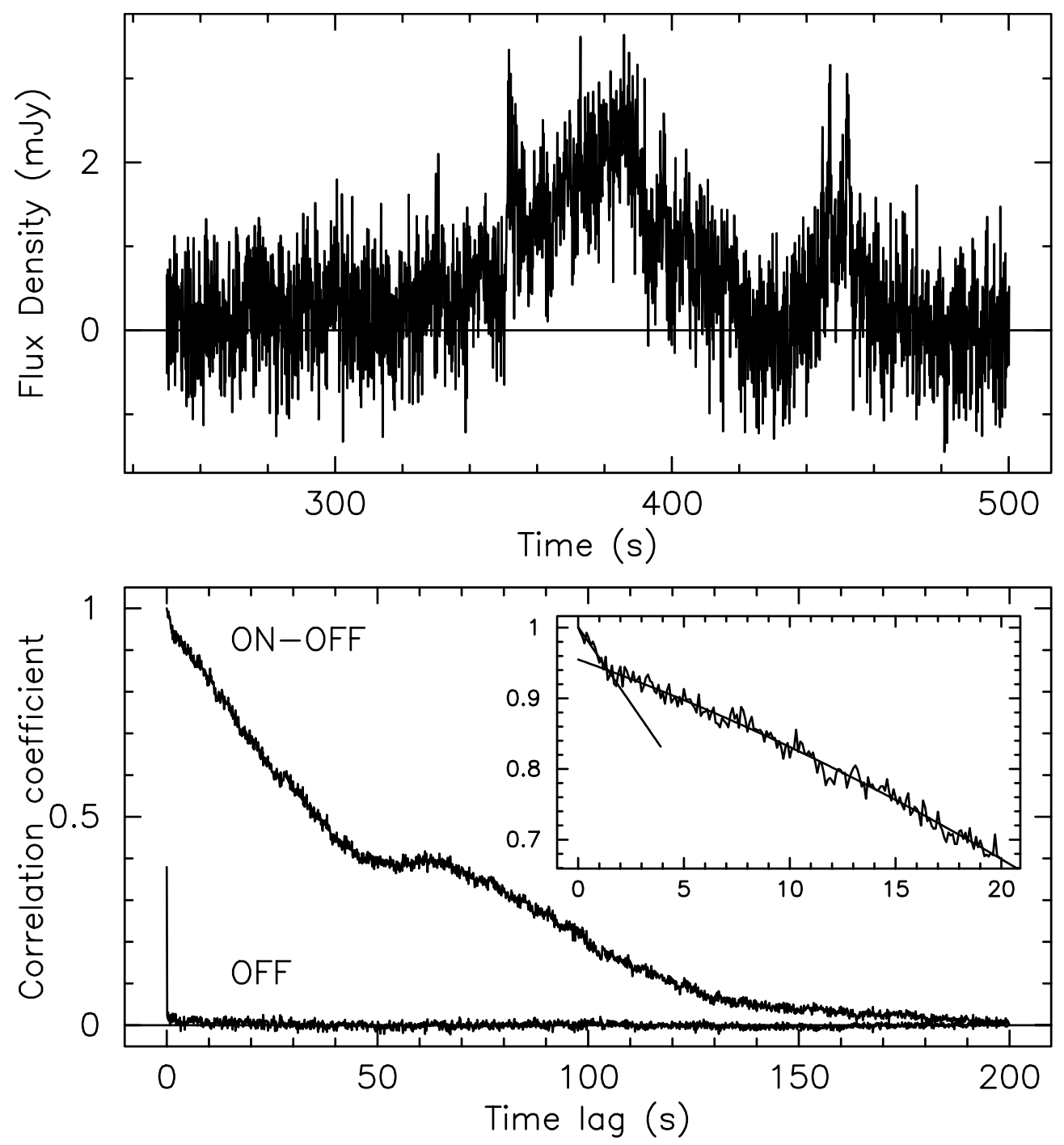

Figure 2. (Top) Bandpass-averaged profile of a circularly polarized flare from J1122+25 observed on 2013 December 27 displayed at the full, $0.1 \mathrm{~s}$ time resolution. (Bottom) A noise-corrected ACF of the flare profile. The inset shows the first 200 lags of the ACF with the solid curves denoting polynomial fits made to emphasize the ACF slope change at the lag of $\sim 2 \mathrm{~s}$. 


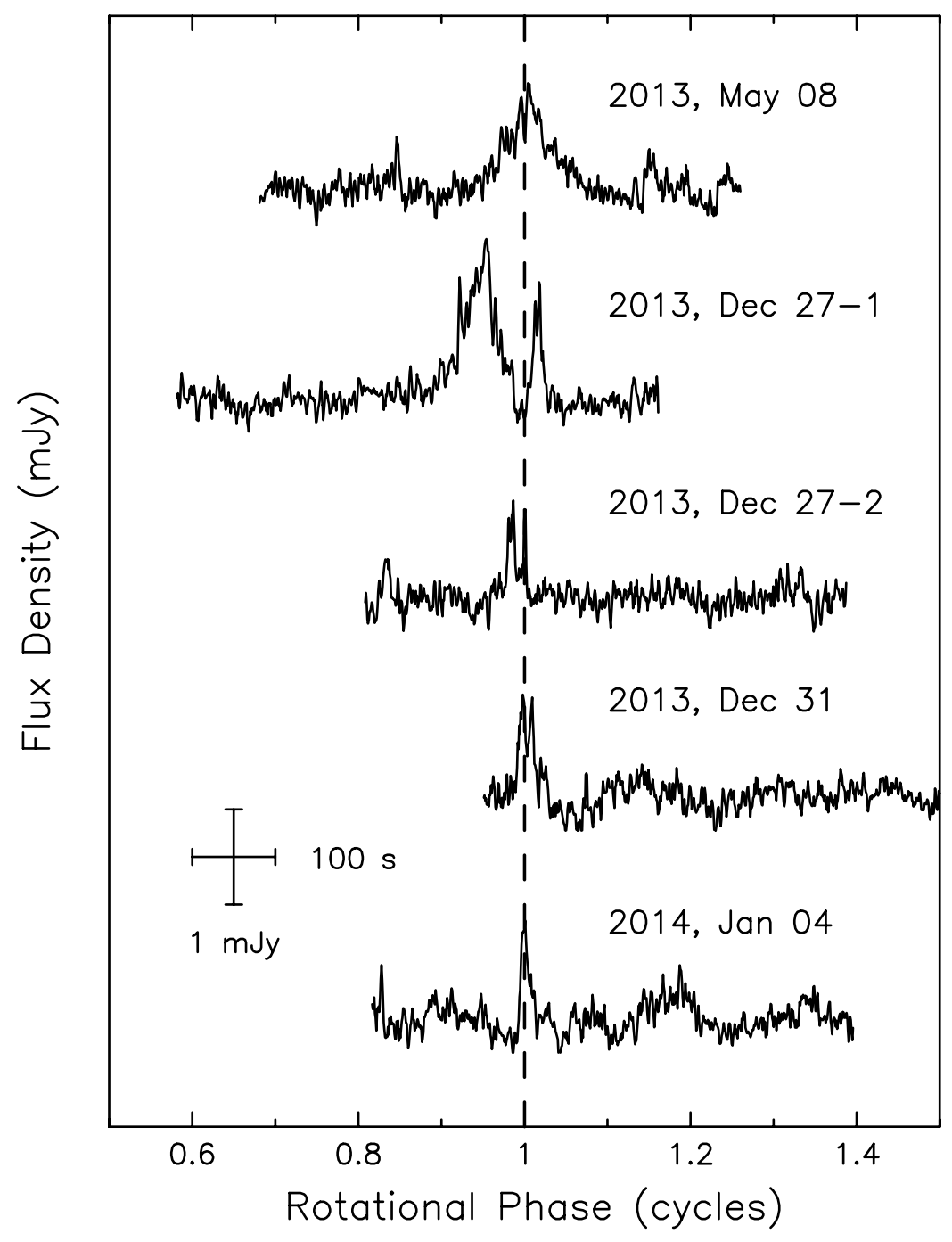

Figure 3. The arrival times of the five flares from J1122+25 observed between 2013 May and 2014 January. The flares are bandpassintegrated, smoothed to the $2.7 \mathrm{~s}$ resolution, and phase-aligned assuming the best-fit, $1035 \mathrm{~s}$ period. The vertical, dashed line marks the arrival times predicted by the model. The actual, best-fit flare arrival times selected by the MC simulation process are: MJD 56421.05858, $56653.39151,56653.40348,56657.35930$, and 56661.35106. 